\title{
Parsed Text Format Versus Regular Block Format in Online Extensive Reading
}

\author{
Leander S. Hughes \\ Saitama University, Saitama, Japan
}

\begin{abstract}
This study investigated the effects of online extensive reading in a syntactically parsed text format compared to regular block format on speed, retention, and proficiency in English reading among Japanese learners of English. A syntactically parsed text format breaks sentences of a text into smaller groups of words or chunks with the intention of increasing the ease of reading. For one academic year, 289 participants at a public university in Japan read English texts of their choosing online in either a parsed format or regular block format. The results showed no significant differences between formats on any of the variables of interest. This suggests that reading in a parsed text format neither hinders nor enhances reading ability or proficiency growth among EFL learners whose first language is linguistically distant from English. Evidence emerged, however, that the reading itself had promoted a significant increase in reading proficiency for both groups.
\end{abstract}

Extensive reading, or the reading of a large quantity of freely chosen texts at or below one's current reading proficiency level, is an effective means of improving English reading proficiency for learners of English as second or foreign language (Nakanishi, 2015). Unfortunately, traditional paper-based extensive reading programs have been difficult to implement in most EFL contexts due to the vast number and variety of reading materials such programs require (Davis, 1995). However, with the steady rise of smartphone and internet use in emerging economies across Asia (Poushter, 2016), extensive reading can now be done online at little or no cost (Lin, 2014), making it a more viable option for a widening range of EFL contexts.

At the same time, viewing texts through a browser rather than off of a printed page allows readers to change the font size, page width and other aspects of the text's format. This new level of control over how a text is displayed raises the question of whether texts could be made more readable through systematically altering their format in some way. Indeed, basic changes in font size, white space, and positioning of a digital text can affect reading performance for both native and non-native readers (Bernard, Chaparro, Mills, \& Halcomb, 2003; Chaparro, Baker, Shaikh, Hull, \& Brady, 2004; Soleimani \& Mohammadi, 2012).

Going beyond conventional format considerations, a number of applications have appeared online which attempt to enhance the readability of digital texts by dramatically altering the way those texts are displayed. One such application is Livelnk.com's WebClipRead (Walker Reading Technologies, Inc., 2016), which uses natural language processing algorithms to display English texts in a syntactically parsed format where sentences are broken into smaller syntactic groups or chunks to improve the ease of reading (Warschauer, Park, \& Walker, 2011).

Language Education in Asia, 2016, 71), 20-31. http://dx.doi.org/10.5746/LEiA/16/V7/I1/A03/Hughes 
The creators of WebClipRead refer to its syntactically parsed text format as visual syntactic text format (VSTF). Figure 1 shows the previous sentence displayed in VSTF.

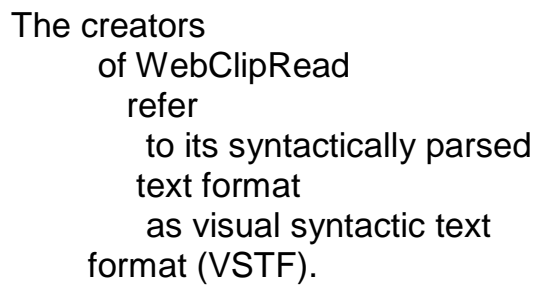

Figure 1. An example of a sentence displayed in visual syntactic text format (Walker Reading Technologies, Inc., 2016).

The use of such a syntactically parsed text format to enhance language learning represents a new direction in EFL. The present study is, to the author's knowledge, the first to investigate whether extensive reading in a parsed text format improves EFL learner's speed, retention, and proficiency in English reading compared to doing the same amount of reading in normal block text format. Though conducted in Japan, this study is relevant for other Asian EFL contextsparticularly emerging economies where internet usage is on the rise-as it investigates the viability of doing extensive reading online as opposed to the traditional and much more expensive paper-based approach.

\section{Literature Review}

\section{Theoretical Basis for Employing a Parsed Text Format}

Displaying texts in a syntactically parsed format such as VSTF may improve reading among learners of English as a foreign language in three ways, by reducing eye regressions, adding virtual prosody to the text, and promoting the acquisition of high-frequency language chunks.

Reducing eye regressions. Reading is rarely a simple linear process. Instead of moving from one word to the next, the reader's eyes frequently backtrack or regress to reread words or find words that were previously skipped over (Brysbaert, Drieghe, \& Vitu, 2005). By limiting the number of words per line, syntactically parsed text formats may decrease the likelihood of losing one's place while reading. The consequent reduction in eye regressions should, in turn, lead to more efficient reading, with lower chances of accidentally skipping over important information (Warschauer et al., 2011).

Adding virtual prosody. Carlson, Frazier, and Clifton (2009) demonstrated the strong relationship between sentence prosody (rhythm, stress, and intonation) and listening comprehension, particularly with regard to syntax. Meanwhile, Kuhn and Stahl (2003) showed how adding prosody to a text can aid reading comprehension. They found that assisted reading, in which learners listen to and shadow a fluent reader's rendition of a text, significantly increased reading proficiency among native remedial learners. Syntactically parsed formats may similarly enhance reading comprehension by breaking and indenting sentences to reveal their internal syntactic boundaries, paralleling the way prosody indicates such boundaries in natural spoken language (Warschauer et al., 2011).

Promoting the acquisition of language chunks. Ellis (2013) provided extensive evidence that language acquisition is a process in which language is constructed from high-frequency formulaic language collocations or chunks. Learners first acquire these chunks and use them intact but gradually learn to manipulate them to create more novel constructions. As learners move from formulaic chunks to more novel expressions, they begin to generalize the syntactic 
patterns shared by the chunks into formal grammar rules (Ellis, 2013). Although, the process of chunk acquisition is largely unconscious, the conscious recognition of common chunks may also play a role (Schmidt, 2001). If so, a syntactically parsed text may directly accelerate language acquisition by clearly demarcating high-frequency language chunks. Moreover, once the learner acquires enough chunks, reading in the parsed format should become more efficient, because the learner can then easily read chunk-by-chunk rather than word-by-word.

\section{Possible Drawbacks}

Parsed text formats may also have some potential drawbacks. First, although shorter line lengths may reduce the number of regressions readers experience, Dyson and Kipping (1998) showed that the corresponding increase in line breaks may slow readers down, hindering fluency. Second, the parsed format may make it difficult to distinguish between paragraphs. This is because parsed formats use indentation and line breaks to display chunks of sentences rather than to demarcate paragraphs as traditional block format does. Parsed formats must therefore use other methods to signal paragraph changes_-VSTF, for example, changes the background color. However, this may feel unnatural to the reader or even result in paragraph changes going unnoticed. Finally, the parsed format is not practical for printed text since it requires many times more pages than block format, so only readers with access to computers or smartphones may benefit from it.

\section{Prior Studies with American Students}

Most studies to date on syntactically parsed text formats have focused on native English readers and have employed WebClipRead's VSTF. Walker, Schloss, Fletcher, Vogel, and Walker (2005) reported on a study involving 40 American university students whose L1 was English. The students read six academic texts, half of which were shown in block format and half in VSTF, with the format for each half randomly determined. Results showed that reading comprehension of texts presented in VSTF was 40 percent higher than that of texts presented in block format. Additionally, readers reported significantly lower eye strain for the texts in VSTF. A more recent study involving 27 American university students compared VSTF to text randomly truncated into lines of the same average length as the lines in VSTF (Warschauer et al., 2011). Again, reading in VSTF resulted in significantly higher comprehension.

Another study reported in Walker et al. (2005) involved 100 American high school students who read their history textbook in class for approximately 50 minutes per week for a year. Half of the students read it on computers in VSTF, while the other half read the conventional paper-based version. Results showed that the VSTF group significantly outperformed the paperbased group both on course quizzes and tests as well as in terms of overall reading proficiency growth. Walker, Gordon, Schloss, Fletcher, Vogel, and Walker (2007) repeated this study using the same design except for having the block format group read their textbooks on computers so that the only difference between groups was the format of the text. Again, the VSTF group achieved significantly higher quiz and test scores as well as overall reading proficiency growth.

While the majority of the participants in the year-long studies reported in Walker et al. (2005) and Walker et al. (2007) were native English speakers, approximately 30 percent of them were non-native English speakers whose L1 was Spanish. Like their native English speaking peers, the non-native participants in the VSTF group also experienced significant gains in test performance and in reading proficiency relative to their counterparts in the block format group. However, unlike the native English participants, who experienced immediate relative gains in quiz scores, it took approximately eight weeks before this difference emerged for the non-native participants. 
The reason for the delay among the non-native participants remains unclear, but the previously described construction grammar approach of Ellis (2013) may hint at a possible explanation. Essentially, native English readers have already acquired the basic chunks that make up much of the target language, so they can take full advantage of the parsed format's chunk-by-chunk presentation of sentences-reading sentences more fluently because those chunks are clearly isolated by the format. On the other hand, non-native readers may not have acquired many of these chunks yet, so they must interpret each line word-by-word, rather than as a chunk. However, as non-native readers acquire more chunks, they gradually become able to read chunk-by-chunk as native English speakers do and a corresponding improvement in reading emerges.

\section{Studies Targeting Non-Native Readers}

Few studies targeting non-native readers have been done on parsed text formats. One of these, by $\mathrm{Yu}$ and Miller (2010), involved 30 Asian non-native English speakers (primarily from Taiwan and China) who read English articles in block text, VSTF, and the researchers' own format, spending approximately 20 minutes reading in each. Results showed no significant difference in reading comprehension for texts in VSTF versus regular block format.

Herbert (2014) had 60 Japanese college students do intensive timed scanning, skimming, and other reading activities using 20 English texts of approximately 200 words each over a threemonth period. The texts were displayed in block format for half of these students and, for the other half, in a syntactically parsed format devised by the researcher to resemble VSTF. After the training period, the two groups were tested on two final texts-one in the parsed format and the other in block format. Results showed no significant differences between groups on any of the skills tested. However, there was a significant difference for all participants in their performance on the final parsed text versus the final block text. On average, participants could find more key words during their timed scanning session in the parsed text than in the block text.

Contrary to Herbert's (2014) expectations though, the majority of the participants who found more key words when reading the parsed text came from the group that had trained on block formatted texts. This result implies that the 20 prior training sessions had no lasting impact on reading ability for the parsed text group and echoes the delay in the emergence of benefits Walker et al. (2005) and Walker et al. (2007) found for their non-native readers.

Parsed text formats represent a highly experimental direction in EFL research. Still, the potential benefits suggested by the American studies justify further investigation. As previous studies seem to show no short term benefits of parsed formats for non-native readers, the present study investigates the longitudinal effects of regular reading in a parsed format for non-native learners of English as a foreign language. Specifically, it addresses the following question:

Does long-term extensive reading in a syntactically parsed text format lead to increased speed, retention, and proficiency in English reading compared to the same amount of reading in regular block format?

\section{Participants}

\section{Method}

This study involved first-year students enrolled in a general English skills course taught by one of eight different teachers at a public university in Japan. Course work included doing extensive reading online as homework. Specifically, students were to read 40,000 words worth 
of English texts of their choosing each semester for two 15-week semesters for 20 percent of their course grade. Students were to do this reading on their computers or mobile phones via AnyRead, an online extensive reading application created by the author. A total of 763 students enrolled in the course and began their extensive reading work. These students were randomized into a syntactically parsed text format group $(n=381)$ and a block format group $(n=382)$. Of these students, 289 completed all of the reading for both semesters. Because this study investigates the effects of long-term regular reading in two different text formats and because no correlation was found between grouping and assignment completion $(r(761)=.02$, $p=.581$, two-tailed), only the data for these 289 participants were included in the final analysis (parsed format group: $n=147$; block format group: $n=142$ ).

\section{Procedure}

As mentioned above, participants did their reading outside of class using AnyRead. At the beginning of the first semester, teachers provided participants with instructions on how to do their extensive reading using AnyRead. These instructions also mentioned that AnyRead would sometimes display texts in an unconventional format and that when it did, participants should just keep reading as they normally do.

Upon creating an AnyRead account, each participant was randomly assigned by the application to either the parsed format or block format group. Randomization was done withinclass to control for differences between teachers and classes. To read in AnyRead, participants simply copied an English text they wished to read from anywhere on the Internet and entered it into the text box in the application. The program would then display the text to read in the assigned format. To display texts in parsed format, the author had previously obtained permission from Walker Reading Technologies, Inc. to allow AnyRead to send the texts directly to the WebClipRead server which formatted them in VSTF and then sent them back to be displayed in that format within AnyRead.

Although participants were aware of being part of a study on reading, they were not aware of their group assignment or even that there were groups. To avoid alerting participants to the existence of the groupings, AnyRead displayed texts in both formats to both groups, but in greatly differing amounts depending on group assignment: the parsed format group read in VSTF 90 percent of the time and block format 10 percent of the time, whereas the block format group read in block format 90 percent of the time and VSTF 10 percent of the time. The researcher and teachers also remained unaware of the group assignments until after the yearlong course had finished, making this study triple blind.

Upon finishing a text, participants clicked a button which led to a retention question (explained further below). If they answered correctly, the number of words they had just read was added to their "Words Read" displayed at the top of their screen. If they answered incorrectly, no words were added and they were not allowed to read the same text again. Students had to increase their words read from 0 to 40,000 each semester for a total of 80,000 words to receive full credit for their reading.

\section{Instruments}

Reading speed and retention were measured via AnyRead. To obtain each participant's mean reading speed, AnyRead measured the time from when each text was displayed until when the participant clicked a button taking them to the retention question. Then it calculated the mean words read per minute (WPM) for all texts read. 
The retention question for each text was generated automatically by AnyRead using a complex algorithm based on the Jist system previously developed and tested by the author (see Hughes, 2013). The question displayed four sentence fragments, each seven to 12 words long. Between one and three of these fragments were extracted verbatim from the text the participant had just read, while the other fragments were taken verbatim or modified from other texts of a similar style from Wikipedia and other sources in the public domain which shared the same key words. To answer correctly, participants had to select the fragments that came from the text they had just read within one minute (see the Appendix for an example question). This was considered a valid method of measuring retention of the content, because the recollection of the ideas and events from the reading was generally necessary for the reader to accurately distinguish between correct and incorrect fragments under time pressure. At the end of the year, overall retention for each participant was then calculated as the percent of retention questions that participants had answered correctly (measuring comprehension would have been preferable to retention, but was technologically infeasible).

To measure participants' English reading proficiency growth, the Test of English for International Communication for Institutional Programs (TOEIC IP) was administered to participants before and after the study period. The researcher would have preferred to use a more widely accepted standardized test, such as the Test of English as a Foreign Language (TOEFL) or the International English Language Testing System (IELTS). However, the TOEIC was employed despite concerns regarding its validity (see Bresnihan, 2012), because it was the only test that the university administers free of charge. Pre-test reading scores were then subtracted from post-test scores to obtain each participant's reading proficiency growth. Of the 289 participants who completed the required amount of extensive reading, 277 took both the pre- and post-TOEIC tests.

\section{Results}

Table 1 displays the descriptive statistics for the reading speed, retention, and proficiency growth of the parsed format and block format groups.

Table 1

Reading Speed, Retention, and Proficiency Growth for Block Versus Parsed Formats

\begin{tabular}{lcccccc}
\hline & \multicolumn{2}{c}{ Speed (WPM) } & \multicolumn{2}{c}{ Retention } & \multicolumn{2}{c}{ Proficiency Growth } \\
\cline { 2 - 6 } Group & Mean & $S D$ & Mean & SD & Mean & $S D$ \\
\hline Block Format & 120 & 32 & 72.1 & 13.3 & 23.9 & 48.5 \\
Parsed Format & 118 & 32 & 72.2 & 12.8 & 19.8 & 52.3 \\
\hline
\end{tabular}

In addition, a two-tailed $t$-test with an alpha level of .05 was conducted for each measure to determine whether any significant differences between groups existed. Table 2 displays the results. 
Table 2

t-Test Results: Parsed Format Means Minus Block Format Means

\begin{tabular}{lccccc}
\hline Measure & Mean Difference & $\begin{array}{c}95 \% \text { Cl for } \\
\text { Mean Difference }\end{array}$ & $d f$ & $T$ & $p$ \\
\hline Speed (WPM) & -2 & $-10,5$ & 287 & -0.64 & .254 \\
$\begin{array}{l}\text { Retention } \\
\begin{array}{l}\text { Proficiency } \\
\text { Growth }\end{array}\end{array}$ & 0.1 & $-2.9,3.1$ & 287 & 0.06 & .956 \\
\hline
\end{tabular}

As shown in Table 2, there were no significant differences between the parsed and block format groups in reading speed, retention, or proficiency growth.

Of note, though, is the reading proficiency growth of both groups as indicated by a paired t-test comparing post TOEIC reading scores $(M=229, S D=67)$ to pre $(M=207, S D=62)$. This revealed a significant mean increase of 22 points, $t(276)=7.20, p<.001,95 \% \mathrm{Cl}[16,28]$. Moreover, there was a significant partial correlation between reading retention and post TOEIC reading scores after controlling for pre TOEIC reading scores, $r(274)=.17, p=.002$, one-tailed. These findings imply that extensive reading significantly increased reading proficiency, particularly for participants who maintained high rates of retention throughout their reading, regardless of their initial proficiency or the format in which they read.

\section{Discussion}

While the amount of reading done appears to have affected reading proficiency growth, no corresponding effect emerged for the parsed text format. The absence of an effect for the parsed text format in this study seems to contradict the results obtained by Walker et al. (2005) and Walker et al. (2007) for their non-native English speaking participants. The non-native participants assigned to the parsed format group in those studies experienced a significant, albeit delayed gain in course quiz scores and after reading for a year in the parsed text format, their reading proficiencies had significantly increased compared to their counterparts in the block text group. Some important differences exist, however, between the non-native learners in these previous studies and the non-native learners in the present study. One is the immersive ESL environment that non-native learners in the previous studies were in. Most notably the L1 of non-native learners in those previous studies was Spanish, whereas the L1 of the participants in the present study was Japanese.

Chiswick and Miller (2005) developed a quantitative measure of the relative amount of difference between English and other languages called linguistic distance. This measure was not based on a comparison of specific phonetic, lexical, or morphological characteristics, but rather on how proficient native English speakers could become at those languages over a given period of time. The lower their average achieved L2 proficiency level, the more linguistically distant their L2 was considered to be from English. The researchers then found that this measurement of the linguistic distances between English and the other languages strongly correlated with the English proficiency levels achieved by immigrants to the United States whose L1s were those respective languages included in their research. Out of the 45 languages analyzed, the researchers found Japanese to be the most linguistically distant from English (along with Korean, followed closely by Cantonese). In comparison, Spanish was less than half as distant from English as Japanese. Thus, perhaps the more linguistically distant one's L1 is from English, the less effective it is to read English in a syntactically parsed text format, or the longer it takes for any benefits to emerge. Recalling Ellis's (2013) construction grammar 
approach, this may be due to the lack of similarity between the high frequency formulaic language chunks of English and those of the distant L1, which make it more difficult and time consuming to acquire enough basic chunks in English for the advantages of the parsed text to emerge. To explore this possibility further, future studies might involve large samples with several L1s at varying linguistic distances from English.

There are other differences that set the present study apart from previous studies. Foremost is this study's within-class randomization and triple-blind design. This design helped guard against the subtle effects of teacher expectation on the performance of the two groups. Rosenthal and Jacobson's (1968) experiment investigating the Pygmalion effect demonstrates just how much teacher expectation can influence student performance, even when both the students and the teacher are unaware of that influence. In the experiment, elementary school teachers were told that a randomly selected group of their students were "late bloomers" or learners who could be expected to show significant intellectual growth in the next year. At the end of the year, the randomly selected students in the first and second grades showed significantly higher IQ test score gains over that year than their peers. The researchers concluded that teachers had unconsciously given preferential treatment to students who had been labelled late bloomers. Similar unconscious influences may have played a role in producing the highly positive results reported in Walker et al. (2005) and Walker et al. (2007). Teachers may have unconsciously given preferential treatment to students in the VSTF group, because teachers expected that reading in VSTF would lead them to do better than their peers. The real effect of the parsed format may thus be weaker than those studies imply.

At the same time, participants in the present study probably read much less than those in the longitudinal studies in Walker et al. (2005) and Walker et al. (2007). In those previous studies, students read a conservatively estimated total of 210,000 words-more than double what participants read in the present study-and they probably read more carefully, because that content was discussed and tested in class. This difference highlights the main weakness of the present study's design, namely the lack of integration between the content students read outside of class and in their in-class activities. This probably lowered their motivation to attend to their reading, which may partially account for the failure of an effect to emerge.

Finally, it is possible that the parsed format did actually have an effect, but that this study's instruments were not sensitive enough to discern it. Indeed, independent attempts to correlate TOEIC scores with other measures of language proficiency have revealed a lower reliability for the test than claimed by its creators (Bresnihan, 2012). The test was sensitive enough, however, to detect the reading proficiency growth expected for both groups due to their extensive reading. This, together with the lack of significant differences between groups on any of the measures used, suggests that the instruments were not at fault.

\section{Practical Implications}

This study has two immediate practical implications for teachers of English in Asian contexts. First, parsed formats may not help Asian EFL learners, most of whom have L1s that are as, or nearly as, linguistically distant from English as Japanese. The second, more positive implication is that having learners do extensive reading using freely available texts online may be a viable and cost-effective way to raise their English reading proficiency in contexts where paper-based English reading resources are limited, but access to the internet is available. Learners would not require any special applications such as AnyRead to engage in extensive reading online. They could simply surf the web and read English texts they find of interest. To monitor learners' reading, teachers could have them write brief reports on what they read and where they read it. For further ideas, see Lin's (2014) thorough review of online extensive reading research. 


\section{Conclusion}

This study investigated whether online extensive reading in a syntactically parsed text format leads to increased reading speed, retention, and proficiency growth compared to reading in regular block text format for learners of English whose first language is linguistically distant from English. Results indicated that, while doing the reading itself seems to have benefitted reading proficiency, no effect emerged for the parsed text format on any of the variables of interest. These findings may imply that the benefits of reading in a parsed text format decrease or take longer to emerge as the linguistic distance between learners' L1 and English increases. At the same time, reading in a parsed text format did not adversely affect speed, retention, or proficiency in English reading. It may be the case then, as Herbert (2014) has suggested, that the proper question to ask is not if reading in a parsed text format is good for EFL learners, but which learners would benefit from it and which would not. Thus, future research might investigate for possible interactions between various individual differences among EFL learners, including the linguistic distance between their L1s and English, and the effects of reading in a syntactically parsed text format. Certainly, further studies in EFL contexts are warranted.

\section{Author Note}

Leander S. Hughes, Saitama University, Saitama, Japan.

Leander Hughes is an associate professor at the Saitama University Center for English Education and Development. He is primarily interested in finding new ways to accelerate language learning. Other research interests include learner autonomy, motivation, and communicative task effectiveness.

This study was supported by a grant from the Japan Society for the Promotion of Science (JSPS Kakenhi Grant Number 26770189). The author would also like to thank the team at Walker Reading Technologies, Inc. (www.liveink.com) for allowing the reading application he created for this study direct unlimited access to the text formatting capabilities of their application WebClipRead.

Correspondence concerning this article should be addressed to Leander S. Hughes, Center for English Education and Development, Saitama University, 255 Shimo-Okubo, Sakura-ku, Saitama City, Saitama 338-8570, Japan. E-mail: hughes@mail.saitama-u.ac.jp 


\section{References}

Bernard, M. L., Chaparro, B. S., Mills, M. M., \& Halcomb, C. G. (2003). Comparing the effects of text size and format on the readability of computer-displayed Times New Roman and Arial text. International Journal of Human-Computer Studies, 59(6), 823-835. http://dx.doi.org/10.1016/S1071-5819(03)00121-6

Bresnihan, B. (2012). Using TOEIC scores to evaluate student performance in English language courses. The Jimbun Ronshu, 43, 1-47.

Brysbaert, M., Drieghe, D., \& Vitu, F. (2005). Word skipping: Implications for theories of eye movement control in reading. In G. Underwood (Ed.), Cognitive processes in eye guidance (pp. 53-77). Oxford, England: Oxford University Press. http://dx.doi.org/10.1093/acprof:oso/9780198566816.003.0003

Carlson, K., Frazier, L., \& Clifton, C., Jr. (2009). How prosody constrains comprehension: A limited effect of prosodic packaging. Lingua, 119(7), 1066-1082. http://dx.doi.org/10.1016/j.lingua.2008.11.003

Chaparro, B., Baker, J.R., Shaikh, A.D., Hull, S., \& Brady, L. (2004, July 12). Reading online text: A comparison of four white space layouts. Usability News. Retrieved from http://usabilitynews.org/

Chiswick, B. R., \& Miller, P. W. (2005). Linguistic distance: A quantitative measure of the distance between English and other languages. Journal of Multilingual and Multicultural Development, 26, 1-11. http://dx.doi.org/10.1080/14790710508668395

Davis, C. (1995). ER: An expensive extravagance? ELT Journal, 49(4), 329-336. http://dx.doi.org/10.1093/elt/49.4.329

Dyson, M. C., \& Kipping, G. J. (1998). The effects of line length and method of movement on patterns of reading from screen. Visible Language, 32, 150-181. http://dx.doi.org/10.1006/ijhc.2001.0458

Ellis, N. C. (2013). Second language acquisition. In G. Trousdale \& T. Hoffmann (Eds.), Oxford handbook of construction grammar (pp. 365-378). Oxford, England: Oxford University Press.

Herbert, J. C. (2014). The effects of syntactically parsed text formats on intensive reading in EFL. The JALT CALL Journal, 10(3), 237-254.

Hughes, L. S. (2013). Investigating the validity and reliability of automatically generated reading comprehension questions. The Journal of Saitama City Educators, 3(6), 6-17. Retrieved from http://www.saitamacityeducators.org/wp-content/uploads/2014/01/Vol.3-Issue6.pdf

Kuhn, M. R., \& Stahl, S. A. (2003). Fluency: A review of developmental and remedial practices. Journal of Educational Psychology, 95, 3-21. http://dx.doi.org/10.1037/00220663.95.1.3

Lauder, S., \& McGregor, W. (June, 2015). Fish and chips. EFLshorts.com. Retrieved from https://eflshorts.com/fish-and-chips/.

Lin, C. (2014). Learning English reading in a mobile-assisted extensive reading program. Computers \& Education, 78, 48-59. http://dx.doi.org/10.1016/j.compedu.2014.05.004

Nakanishi, T. (2015). A meta-analysis of extensive reading research. TESOL Quarterly, 49(1), 637. http://dx.doi.org/10.1002/tesq.157

Poushter, J. (2016, February 22). Smartphone ownership and internet usage continues to climb in emerging economies. Pew Research Center. Retrieved from http://www.pewglobal.org/2016/02/22/smartphone-ownership-and-internet-usagecontinues-to-climb-in-emerging-economies/

Rosenthal, R., \& Jacobson, L. (1968). Pygmalion in the classroom. New York, NY: Holt, Rinehart \& Winston. 
Schmidt, R. (2001). Attention. In P. Robinson (Ed.), Cognition and second language instruction (pp. 3-32). Cambridge, England: Cambridge University Press. http://dx.doi.org/10.1017/CBO9781139524780.003

Soleimani, H., \& Mohammadi, E. (2012). The effect of text typographical features on legibility, comprehension, and retrieval of EFL learners. English Language Teaching, 5(8), 207-216. http://dx.doi.org/10.5539/elt.v5n8p207

Walker, R. C., Gordon, A. S., Schloss, P., Fletcher, C. R., Vogel, C., \& Walker, S. (2007). Visualsyntactic text formatting: Theoretical basis and empirical evidence for impact on human reading. Paper presented at the IEEE Professional Communication Conference, 2007, Seattle. 1-14. http://dx.doi.org/10.1109/IPCC.2007.4464068

Walker Reading Technologies, Inc. (2016). WebClipRead [Computer software]. Available at http://www.liveink.com/

Walker, S., Schloss, P., Fletcher, C. R., Vogel, C. A., \& Walker, R. C. (2005). Visual-syntactic text formatting: A new method to enhance online reading. Reading Online, 8(6). Retrieved from http://www.readingonline.org/

Warschauer, M., Park, Y., \& Walker, R. (2011). Transforming digital reading with visualsyntactic text formatting. The JALT CALL Journal, 73), 255-270.

Yu, C. S., \& Miller, R. C. (2010). Enhancing web page readability for non-native readers. In E. D. Mynatt, D. Schoner, G. Fitzpatrick, S. E. Hudson, W. K. W. Edwards, \& T. Rodden (Eds.), Proceedings of ACM CHI 2010 Conference on Human Factors in Computing Systems, 2010, 2523-2532. http://doi.acm.org/10.1145/1753326.1753709 


\section{Appendix}

\section{An Example of an AnyRead Retention Question}

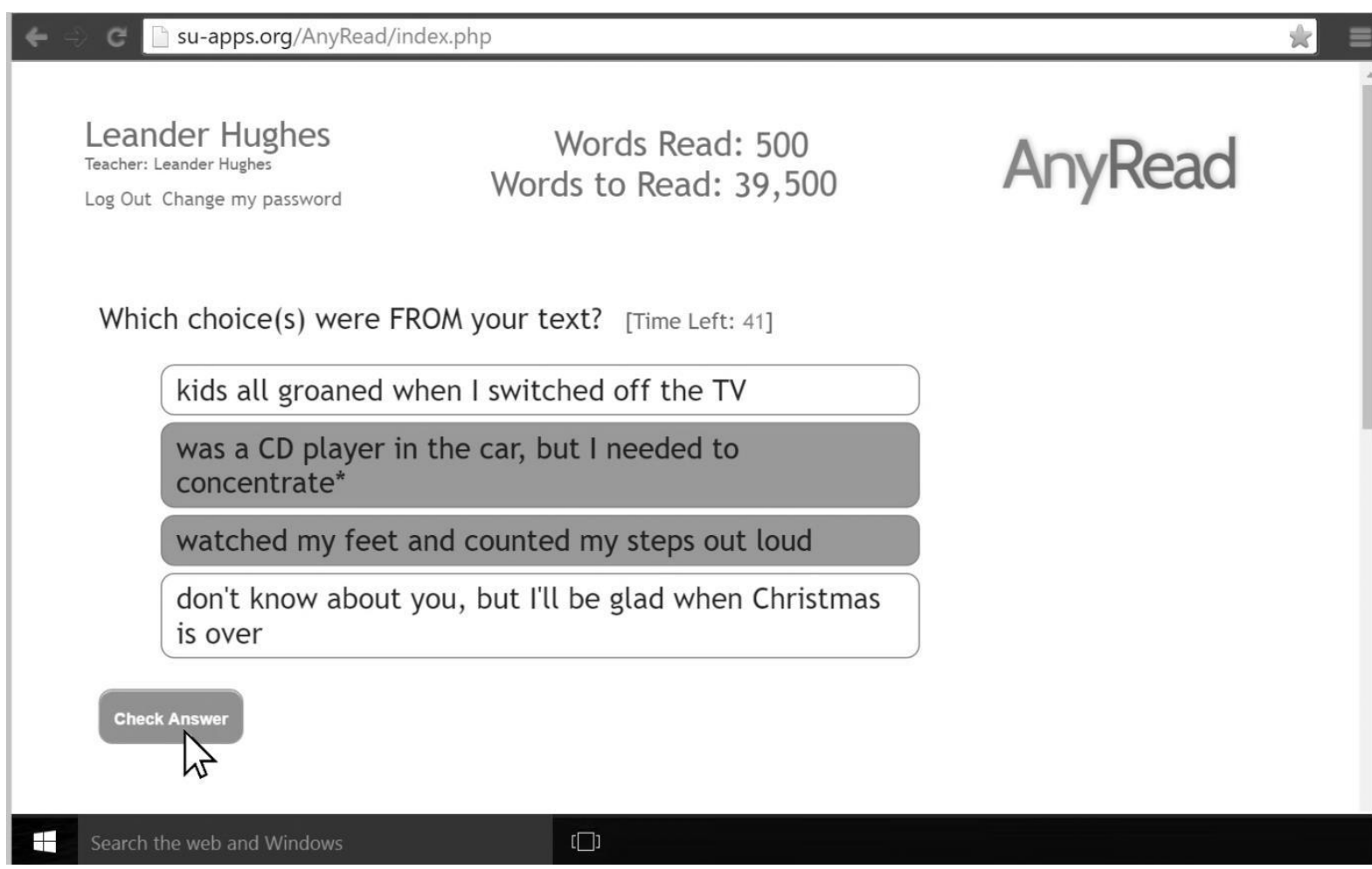

This question was generated for the first two chapters of the story "Fish and Chips" (Lauder \& McGregor, 2015). The correct choices, which came verbatim from that text, are shown in green when the user clicks the "Check Answer" button. 